\title{
THE OCTOBER MEETING IN PHILADELPHIA
}

The six hundred thirty-seventh meeting of the American Mathematical Society was held at the University of Pennsylvania in Philadelphia, Pennsylvania, on Saturday, October 29, 1966. About 102 persons attended, including 85 members of the Society.

By invitation of the Committee to Select Hour Speakers for Eastern Sectional Meetings, Professor Raoul Bott of Harvard University addressed the Society on The Riemann-Roch question in various guises. Professor William Browder presided and introduced the speaker.

There were three sessions for seventeen contributed papers on Saturday morning and afternoon. Professors Wendell Fleming, E. E. Floyd, and Paul Kumpel were the session chairmen.

Everett Pitcher Associate Secretary

Bethlehem, Pennsylvania 